\title{
Comparison of locomotor pattern between idiopathic scoliosis patients and control subjects
}

\author{
François Prince*, Mathieu Charbonneau, Geneviève Lemire, Charles-Hilaire Rivard \\ From 7th International Conference on Conservative Management of Spinal Deformities \\ Montreal, Canada. 20-22 May 2010
}

\begin{abstract}
Introduction
Idiopathic scoliosis has no known cause. One the potential hypothesis is the influence of an asymmetrical nervous command on the muscles acting the spine. The aim of this study is to compare idiopathic scoliosis patients (ISP) and control adolescents (CTRL) during locomotion at comfortable speed. The specific purposes of this study are to compare both groups during walking 1) for EMG activity of the erector spinea muscles and the kinematics (acceleration) of the spine in the antero-posterior (A/P) direction and 2) for the kinetics (moment, power and work) at the ankle, knee and hip.
\end{abstract}

\section{Material and methods}

A total of nine ISP and ten control subjects were asked to walk for 10 trials on a $10 \mathrm{~m}$ walkway. The ISP had a mean Cobb angle of $17^{\circ}$ and their mean age, height and weight were $14,9 \mathrm{y}, 158 \mathrm{~cm}$ and $51,5 \mathrm{~kg}$ respectively while it was $14,4 \mathrm{y}, 161,2 \mathrm{~cm}$ and $55 \mathrm{~kg}$ for the CTRL group. A total of 30 reflective makers placed on anatomical landmarks were tracked with by 8-camera VICON system while muscle activation were collected from eight EMG electrodes placed on the erector spinea muscle at $\mathrm{C} 7$, T4, T10 and L2 levels. Two embedded force platforms were used to extract the ground reaction forces. Moment of force (N.m), power (W) and mechanical energy $(\mathrm{J})$ were calculated from an inverse dynamic approach. Group differences were assessed with analysis of variance (ANOVA) and a level of significance of 0,05 was chosen.

\section{Results}

No difference was observed in the gait velocity between both groups. The ISP group had a greater delay of

Sainte-Justine Hospital, Montreal, Canada

Full list of author information is available at the end of the article muscle activation at $\mathrm{C} 7, \mathrm{~T} 4$ and $\mathrm{T} 10$ with respect to $\mathrm{L} 4$ level compared to the CTRL group. The A/P accelerations were damped from $\mathrm{S} 1$ to the head while not much vertical acceleration damping was observed between these levels. For the kinetics, no difference was observed for the moment of force and power peaks. For the work parameters, the ankle plantar flexor (A2) and hip flexor (H3) work were significantly reduced in the in ISP compared to the CTRL group. Bilateral (right vs. left) significant difference was observed for the knee absorption burst (K1) by the knee extensors.

\section{Discussion and conclusion}

The results suggested the existence of a possible asymmetrical nervous command for the knee extensor during walking in ISP. A delay of spinal muscle activation was observed in the ISP group. The trend for a reduced in locomotion speed in ISP is linked with a reduction energy generation of the ankle and hip at push and pulloff.

Published: 10 September 2010

doi:10.1186/1748-7161-5-S1-034

Cite this article as: Prince et al:: Comparison of locomotor pattern

between idiopathic scoliosis patients and control subjects. Scoliosis 2010 5(Suppl 1):O34. 